\title{
A New Method of Image Denoising for Underground Coal Mine Based on the Visual Characteristics
}

\author{
Gang Hua ${ }^{1}$ and Daihong Jiang ${ }^{1,2}$ \\ ${ }^{1}$ School of Information and Electrical Engineering, China University of Mining and Technology, Xuzhou, Jiangsu 221008, China \\ ${ }^{2}$ School of Information and Electronic Engineering, Xuzhou Institute of Technology, Xuzhou, Jiangsu 221008, China
}

Correspondence should be addressed to Gang Hua; ghua3323@163.com

Received 15 January 2014; Accepted 12 March 2014; Published 6 April 2014

Academic Editor: Feng Gao

Copyright (C) 2014 G. Hua and D. Jiang. This is an open access article distributed under the Creative Commons Attribution License, which permits unrestricted use, distribution, and reproduction in any medium, provided the original work is properly cited.

\begin{abstract}
Affected by special underground circumstances of coal mine, the image clarity of most images captured in the mine is not very high, and a large amount of image noise is mingled with the images, which brings further downhole images processing many difficulties. Traditional image denoising method easily leads to blurred images, and the denoising effect is not very satisfactory. Aimed at the image characteristics of low image illumination and large amount of noise and based on the characteristics of color detail blindness and simultaneous contrast of human visual perception, this paper proposes a new method for image denoising based on visual characteristics. The method uses CIELab uniform color space to dynamically and adaptively decide the filter weights, thereby reducing the damage to the image contour edges and other details, so that the denoised image can have a higher clarity. Experimental results show that this method has a brilliant denoising effect and can significantly improve the subjective and objective picture quality of downhole images.
\end{abstract}

\section{Introduction}

The application environment in the coal industry is always special, and downhole images are always mingled with large amount of image noise interfered by complex underground environment, mechanical vibration, and dust noise. This brings many difficulties for the subsequent processing of the image. Therefore, the denoising process on images captured and transmitted from downhole becomes very necessary so as to provide better image quality and better followup processing.

There are many ways in terms of denoising, such as the mean denoising, median denoising, and wavelet denoising. A representative study is from Narendra, who raised the row-column decomposition median filtering algorithm [1]; moreover, He et al. have put forward a multimedian filter algorithm [2] which can effectively remove the image impulse noise; additionally, Darsow and Olsen have proposed three denoising methods based on phases of wavelet: local phase variance threshold, edge tracking, and scale phase fluctuation threshold method [3]. In order to overcome the weakness that wavelet transform can only carry out point singularity detection, Minh N. Do and Martin Vetterli proposed contourlet transform in 2002 [4]. With the gradual deepening of the various branches of mathematics in the theory and applications, great progress in image denoising technology has been achieved in terms of mathematical morphology, partial differential equations, genetic algorithms, information theory, and so forth, producing a number of new denoising algorithms [5-8], including denoising algorithm based on mathematical morphology [9-12], denoising algorithm based on fuzzy theory $[13,14]$, denoising algorithm based on genetic algorithms [15], neural network-based denoising algorithm [16], and denoising algorithm based on information entropy.

Although, with the maturity and improvement of the various theories, image denoising methods have gained a lot of progress; these methods have their respective advantages, disadvantages, and application areas. For example, the mean denoising is suitable for removing grain noise in images, but the images always easily become blur because this method is too average; median denoising is good for removing impulse noise in the image, but the denoising effect is not very ideal when the noise area inside the window is too large; Wiener filter is suitable for removing the white 
noise in the image; however, the calculation amount is too large; wavelet denoising has a wide range of algorithms and superior performances, but the realization is very difficult and complex. Moreover, the effect of the above algorithms will not be satisfactory when an image is simultaneously interfered by a variety of noise; in other words, while the image smoothens, the quality declines. Besides, denoising research on specific areas with complex environment is also very rare. The aforementioned are the current research status of image denoising; therefore, with the combination of noise characteristics of the coal mine, finding a method that can preserve the image detail and textural features while at the same time reducing image noise has become the research goal of this paper.

\section{CIELab Color Space}

CIELab color space is defined by the International Commission on Illumination (CIE) in 1976, and it is currently one of the most uniform color spaces with a set data of $L, a$, and $b$ representing one color, and one Lab values group formed one corresponding relationship with one color. In the set, $L$ indicates the luminance value; $a$ and $b$ are the chromaticity coordinates. Value $a$ indicates the color change direction of red-green, $+a$ indicates the change towards the red direction, and $-a$ shows the change in the direction of green. $b$ shows the change in the yellow-blue direction, $+b$ shows the change in the direction of yellow, and $-b$ shows the change in the blue direction. As shown in Figure 1, $a$ represents axis of red and green and $b$ represents axis of yellow and blue. Their values range from 0 to 10. $a=b=0$ means colorlessness, and $L$ represents scale factor from black to white.

\section{Interconversion of RGB and CIELab Color Space}

The interconversion of RGB and CIELab color space needs to convert RGB to CIEXYZ color space first and then to CIELab color space [5].

The conversion formula of RGB color space to $X Y Z$ color space is

$$
\left[\begin{array}{l}
X \\
Y \\
Z
\end{array}\right]=\left(\begin{array}{lll}
0.4303 & 0.3416 & 0.1784 \\
0.2219 & 0.7068 & 0.0713 \\
0.0202 & 0.1296 & 0.9393
\end{array}\right)\left[\begin{array}{l}
R \\
G \\
B
\end{array}\right]
$$

Then, convert $X Y Z$ color space to LAB color space, and the conversion formula is

$$
\begin{gathered}
a=500\left[f\left(\frac{X}{X_{n}}\right)-f\left(\frac{Y}{Y_{n}}\right)\right], \\
b=200\left[f\left(\frac{Y}{Y_{n}}\right)-f\left(\frac{Z}{Z_{n}}\right)\right], \\
f(x)= \begin{cases}x^{1 / 3} & \frac{Y}{Y_{n}}>0.008856, \\
7.787 \times x+\frac{16}{116} & \frac{Y}{Y_{n}} \leq 0.008856,\end{cases}
\end{gathered}
$$

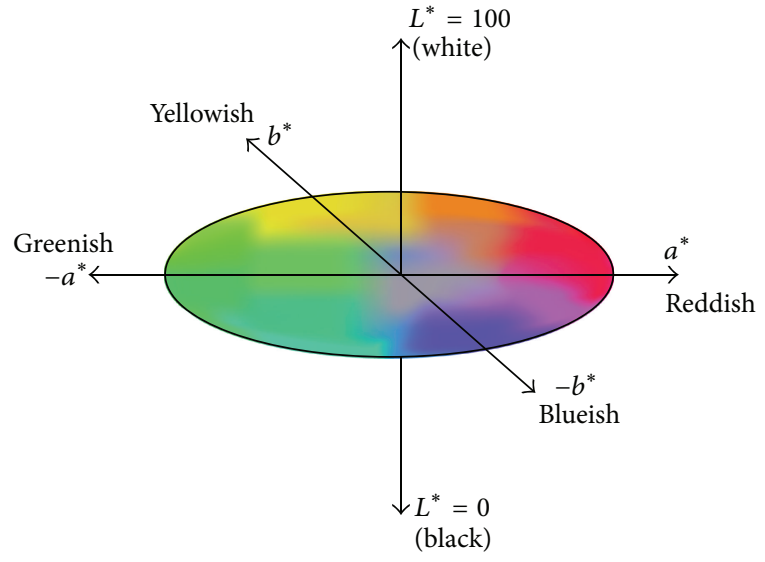

Figure 1: CIELab color space.

where $L=f(x), X_{n}=95.04, Y_{n}=100.00$, and $Z_{n}=$ 108.89 are white tristimulus values of $D_{65}$, the CIE standard illuminant; $X, Y$, and $Z$ are coordinate value of CIEXYZ space.

The computational formula of aberration $\Delta E_{a b}^{*}$ between two colors in CIEXYZ space is as follows:

$$
\Delta E_{a b}=\left[\left(\Delta L^{*}\right)^{2}+\left(\Delta a^{*}\right)^{2}+\left(\Delta b^{*}\right)^{2}\right]^{1 / 2},
$$

where

$$
\begin{aligned}
& \Delta L^{*}=L_{1}^{*}-L_{2}^{*}, \\
& \Delta a^{*}=a_{1}^{*}-a_{2}^{*}, \\
& \Delta b^{*}=b_{1}^{*}-b_{2}^{*} .
\end{aligned}
$$

\section{New Adaptive Image Denoising Method Based on Visual Characteristic}

The classic image denoising methods are done in the RGB color space, while RGB color space is a nonuniform color space, and it does not take into account important information such as the image brightness and chroma. Due to the fact that images captured from downhole are affected by low light or uneven illumination, it is very hard to reach satisfactory denoising effect by adopting the classic image denoising method. In order to improve the image denoising performance, a more uniform CIELab color space is needed. In this color space, human visual sensitivity to the color differences of different wavelengths is not the same; the relevant data are shown in Table 1. Aimed at the special downhole circumstances and based on CIELab uniform color space, the characteristics of color detail blindness, and simultaneous contrast of human visual perception, this paper presents a new image denoising method to improve the subjective and objective image quality.

4.1. Algorithm Thought. In uniform color space, the chromatism value of two human-eye distinguishable colors is equal; that is, when the chromatism is smaller than a certain 
TABLE 1: Value of chromatism and sensitivity level to the color differences.

\begin{tabular}{ll}
\hline $\begin{array}{l}\text { Value of } \\
\text { chromatism }\end{array}$ & Sensitivity level to the color differences \\
\hline $0.0 \sim 0.50$ & (Tiny chromatic aberration) trivial feeling \\
$0.5 \sim 1.51$ & (Small chromatic aberration) slight feeling \\
$1.5 \sim 3$ & (Lesser chromatic aberration) noticeable feeling \\
$3 \sim 6$ & (Larger chromatic aberration) appreciable feeling \\
Above 6 & (Large chromatic aberration) strong feeling \\
\hline
\end{tabular}

threshold, human eyes consider them as the same color; but when the chromatism is greater than a threshold, human eye will be able to distinguish the two different colors. In CIELab color space, the value of this threshold is generally 3 . In view of this, the paper will divide image noise into two types: flat region noise and nonflat region noise. The algorithm carries out different processing self-adaptively according to the different region of the pixel.

In a region, let $x_{i j}$ represent the polluted pixel value of point $(i, j)$, and then let the CIELab median of the pixel in the area with $x_{i j}$ being the center point and neighborhood being $(2 N+1) \times(2 N+1)$ be

$$
y_{i j}=\text { median }=\left\{x_{i-N, J-n}, \ldots, x_{i j}, \ldots, x_{i+N, J+N}\right\} .
$$

Let the CIELab chromatic aberration of current pixel point and center point be $d=\left|x^{i j}-y^{i j}\right|$; preset a threshold $t$, and compare it with $d$ :

$$
\alpha_{i j}= \begin{cases}1 & d>t \\ 0 & \text { else. }\end{cases}
$$

If $\alpha_{i j}$ of all pixels within the neighborhood of $(2 N+1) \times(2 N+$ 1) are 1, then, it is called flat region noise; the color of flat region noise point can be affected by the rest of pixels within the same neighborhood, and convolution denoising can be conducted by using traditional Gaussian filter template. If there are pixels within the neighborhood of $(2 N+1) \times(2 N+$ $1)$ whose $\alpha_{i j}$ are 0 ; then, this area is nonflat region. Noise in nonflat region is not entirely affected by other pixels in the neighborhood and is only related to pixels in the same area; that is, the noise is only related to pixels whose CIELab chromatic aberration is less than the relevant threshold; if it is greater than the threshold, then it is considered that the contribution of the pixel color value to the center point remains conforming to Gaussian distribution; otherwise, the pixel does not have any contribution to the center point. Let the convolution weights be 0 . Thus, the pixel color will be more consistent within the same region, achieving the purpose of preserving image detail.

4.2. Gaussian Filter. Gaussian filter [7] is the linear smoothing filter which determines weights according to the shape of the Gaussian function. One-dimensional Gaussian function is

$$
g(x)=e^{-x^{2} / 2 \sigma^{2}}
$$

wherein $\sigma$ determines the width of the Gaussian filter. The greater $\sigma$ becomes, the wider the band of the Gaussian filter will be and the better the smoothness will be. Moreover, $a$ compromise can be obtained by adjusting the smoothness degree parameter $\sigma$, when the image features are too vague and when excessive unwelcome break variables are caused by noise and texture in smooth images. For image processing, two-dimensional Gaussian function is commonly used as smoothing filter. The function expression is

$$
g[i, j]=e^{-\left(i^{2}+j^{2}\right) / 2 \sigma^{2}} .
$$

The convolution and denoising formula of the input image $f[i, j]$ via Gaussian filter is

$$
\begin{aligned}
g[i, j] * f[i, j] & =\sum_{k=0}^{m-1} \sum_{l=0}^{n-1} g[k, l] f[i-k, j-l] \\
& =\sum_{k=0}^{m-1} \sum_{l=0}^{n-1} e^{-\left(k^{2}+l^{2}\right) / 2 \sigma^{2}} f[i-k, j-l] \\
& =\sum_{k=0}^{m-1} e^{-k^{2} / 2 \sigma^{2}}\left\{\sum_{l=0}^{n-1} e^{-l^{2} / 2 \sigma^{2}} f[i-k, j-l]\right\} .
\end{aligned}
$$

In order to reduce the time complexity of Gaussian filter convolution calculation, the two-dimensional Gaussian function can be converted to the combination of two onedimensional convolution templates [8]. First, convolve the input image $f[i, j]$ and the Gaussian template in the horizontal direction; set a temporary array; store the result in the temporary array; then convolve the image and the Gaussian template in the horizontal direction and transpose the result; the final smooth image can be obtained.

Figure 2 is a schematic diagram of the separability of the Gaussian function convolution. This method is completed through the combination of two horizontal convolution templates. First, convolve the input image $f[i, j]$ and the Gaussian function in the horizontal direction, and then transpose and store the result in the temporary array; after that, take the temporary array as the input, and carry out the convolution with the same Gaussian function to realize the purpose of replacing the vertical convolution with the horizontal convolution. Transpose the output information after the second convolution, and then the final smoothed output image can be obtained. Since the separability of the Gaussian function is high, Gaussian filter can be effectively achieved. Two-dimensional Gaussian function can be carried out in two steps. First, convolve the image with the one-dimensional Gaussian function; then, convolve the result with the same one-dimensional Gaussian function perpendicular to the first result. Therefore, the calculation amount of two-dimensional Gaussian filter presents a linear growth along with the width of the filter template rather than a square growth.

4.3. Algorithm Flow. Define the size of denoising template as $\delta \times \delta$ and the distinguishable color threshold as $T$; traverse any pixel $(r, g, b)$ in the image; the specific realization steps of this algorithm are as follows. 


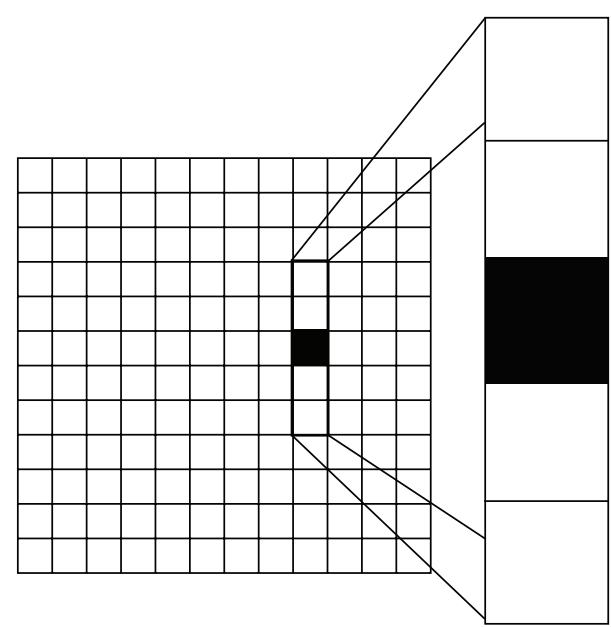

(a) Longitudinal template convolution

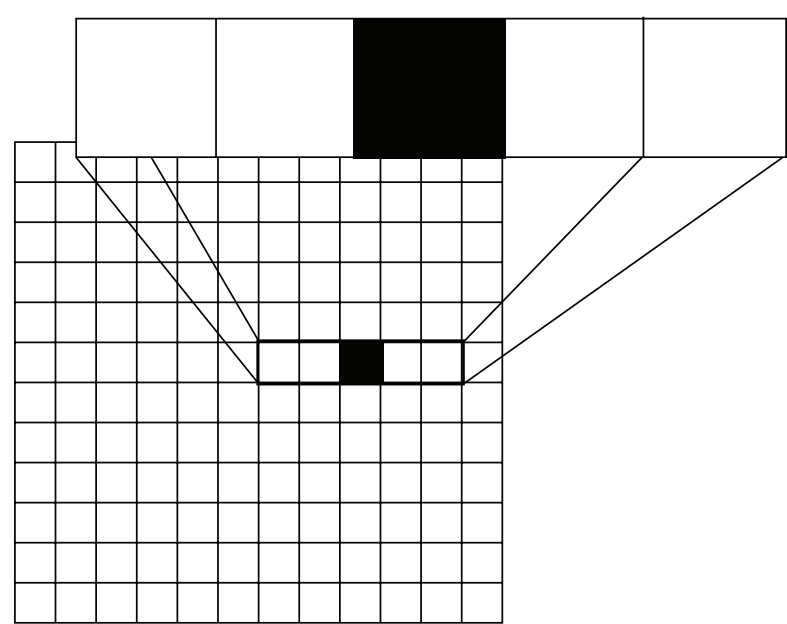

(b) Horizontal template convolution

FIGURE 2: Schematic diagram of the separability of Gaussian function convolution.

(1) Input the pending image $f$ and initialize the Gaussian convolution template whose size is $\delta \times \delta$.

(2) Use the chromatic aberration computational formula of CIELab space color to calculate the value of chromatism between the center pixel and pixels within neighborhood $\delta$; collect number $n$ which stands for the value of chromatism that is greater than the threshold $T$.

(3) If $n=\delta \times \delta-1$, then it indicates that except the center pixel all the values of chromatism between any two pixels in the region are greater than the threshold $T$. This is flat region noise; convolution and denoising can be reached via traditional Gaussian filter template. Turn to Step (4).

(4) If $n \neq \delta \times \delta-1, n \neq 0$, and if the value of chromatism between center pixel and pixel $(i, j)$ in the region is greater than threshold $T$, then this is nonflat region noise. Set the value of pixel $(i, j)$ in Gaussian convolution template to be 0 . Turn to Step (5).

(5) If none of the above is satisfied, no operation is required; keep the original value.

(6) Process the next pixel and turn to Step (2).

(7) Judge whether all the pixels are processed; if yes, end the algorithm; otherwise, turn to Step (6) and continue the processing.

The algorithm flow is as shown in Figure 3.

\section{Experimental Performance and Comparative Analysis}

A noisy downhole image was selected and denoising processing was carried out by, respectively, using methods of mean denoising, median denoising, hybrid denoising, traditional

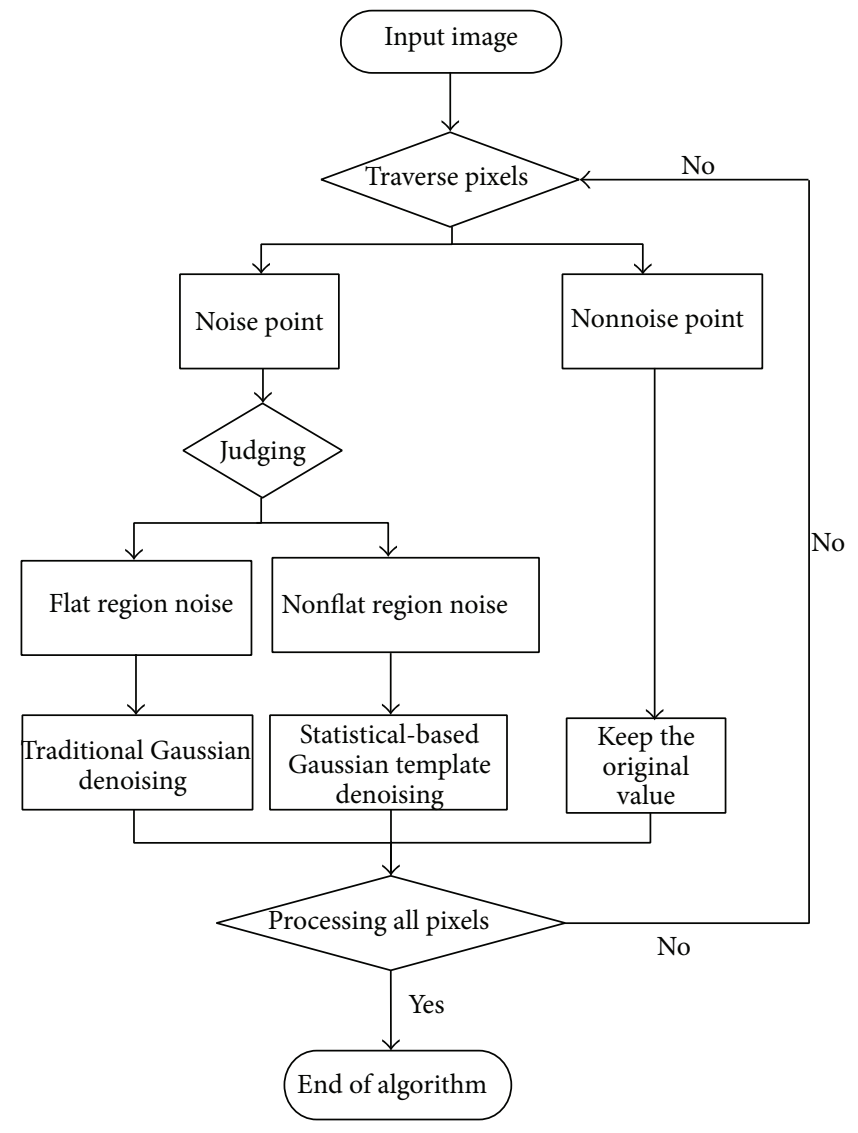

FIGURE 3: Algorithm flow.

Gaussian denoising, and the method proposed in this paper. Image RMSE (root mean square error) and PSNR (peak signal to noise ratio) were calculated, so as to evaluate the performance of different algorithms. 


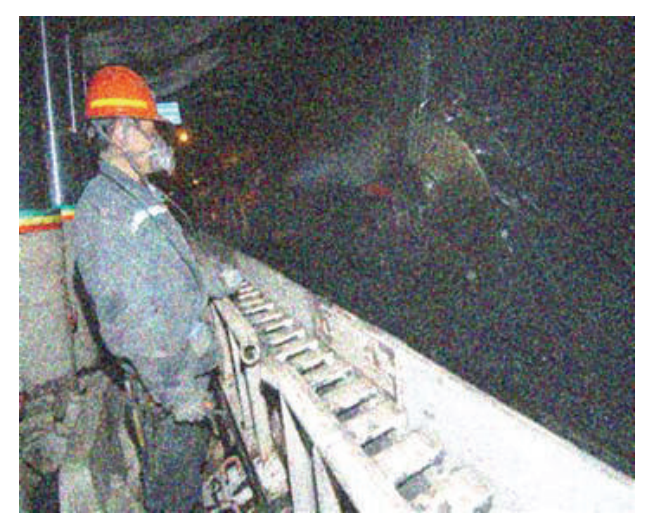

(a) Noise polluted image

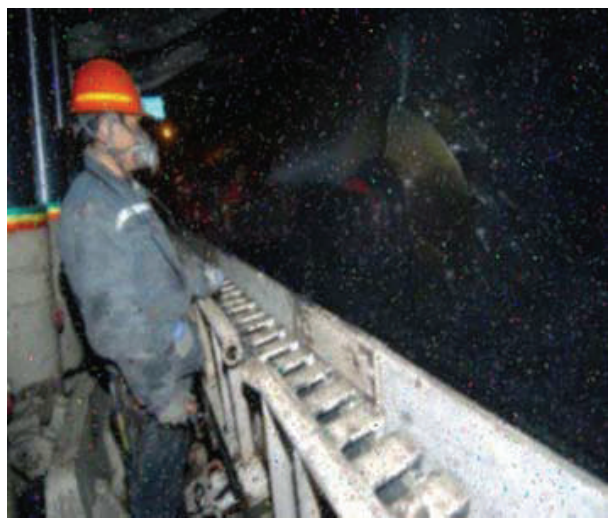

(c) Median denoising

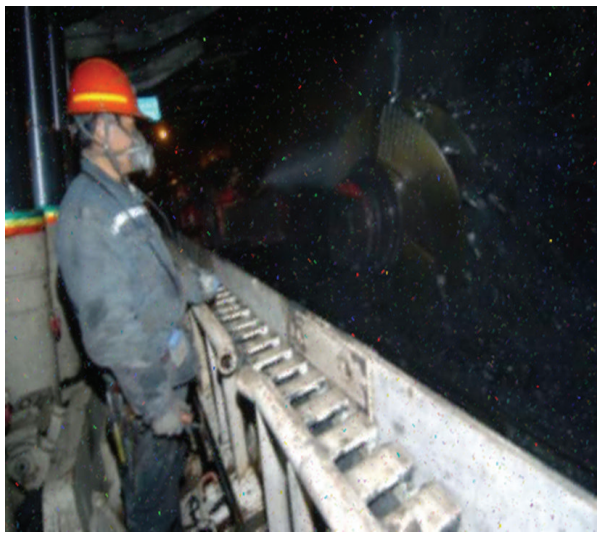

(e) Traditional Gaussian denoising

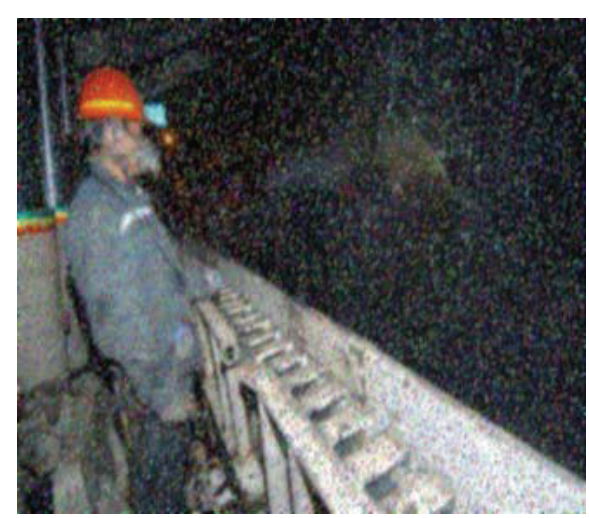

(b) Mean denoising

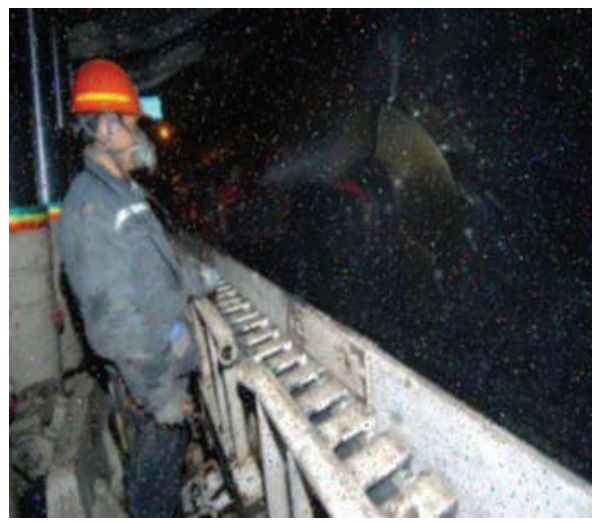

(d) Hybrid denoising

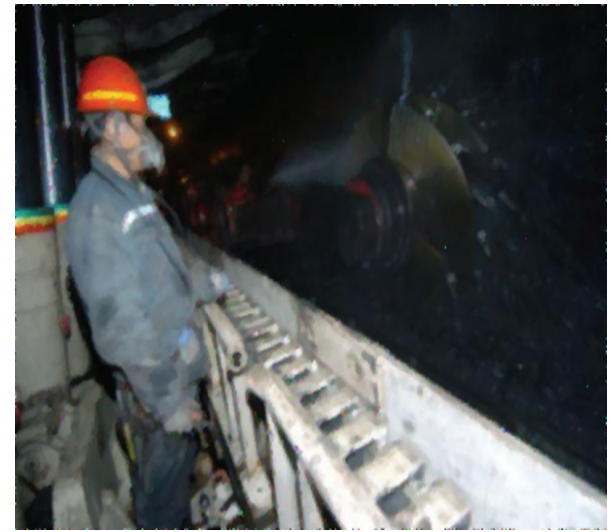

(f) Paper-proposed denoising

FIGURE 4: Effect contrast of different denoising methods.

RMSE and PSNR are defined as follows $[17,18]$ :

$$
\begin{aligned}
& \mathrm{RMSE}=\frac{1}{M N} \sum_{j=1}^{N} \sum_{i=1}^{M}|f(x, y)-g(x, y)|^{2} \\
& \mathrm{PSNR}=10 \log _{10}\left(\frac{255^{2} \times M \times N}{\sum_{j=1}^{N} \sum_{i=1}^{M}(f(i, j)-g(i, j))^{2}}\right),
\end{aligned}
$$

wherein $g$ indicates source image, $f$ stands for images which goes through scales addressing first and then same multiple processing second with the use of relevant algorithm, and $M$ and $N$ represent the length and width of the image. RMSE reflects the approximate extent of the scaled image to the source image; the smaller the RMSE is, the more approximate the scaled image is to the source image; PSNR reflects the image magnification effect. The higher the PSNR is, the clearer the scaled image will be.

The experimental hardware environment is Pentium 4 CPU $(2.80 \mathrm{GHz})$, memory capacity $1.5 \mathrm{~GB}$, and resolution $1024 \times 768$; the software environment is Microsoft Windows XP Professional SP3 operating systems. The effect contrast of 
TABLE 2: Evaluation index of denoising effect of different denoising algorithms.

\begin{tabular}{lcc}
\hline Denoising method & RMSE & PSNR \\
\hline Mean denoising & 307.14593 & 21.03142 \\
Median denoising & 259.65736 & 32.73148 \\
Hybrid denoising & 242.47657 & 33.45765 \\
Traditional Gaussian denoising & 210.32459 & 34.87167 \\
Paper-proposed denoising & 190.10174 & 36.91472 \\
\hline
\end{tabular}

different denoising methods is as shown in Figure 4, and the comparison of denoising experimental data is as shown in Table 2.

It can be seen from Table 2 that RMSE of mean denoising is the maximum, and that of the PSNR is minimum, which means the denoising effect of this method is the most unsatisfactory. RMSE of median and hybrid denoising method is greatly lower than that of mean denoising, and PSNR has also been improved. Compared with traditional Gaussian denoising and other methods, the algorithm proposed by this paper has further improved the objective quality of the image. Moreover, it can also be seen from Figure 3 that the contours of the image processed by mean denoising are vague, and the image also contains a lot of noise; what is worse, there is a serious loss of image detail information; the performance of median and hybrid denoising is a little bit better, but the processed image still contains residual noise; traditional Gaussian denoising method did not manage to eliminate the obvious noise within the visual range, and the effect is not very ideal; the algorithm proposed in this paper successfully improved the peak signal to noise ratio and at the same time managed to reserve image detail features and texture changing features well; more importantly, the processed image has higher resolution and better visual effects.

\section{Conclusions}

Denoising processing on underground images has been conducted in this paper based on CIELab color space and CIELab chromatism aberration computational formula. The paper has proposed that weight should be determined by visual chromatism aberration, which makes up the insufficiency of letting space coordinates distance decide weight in RGB space; in the meantime, the characteristic that CIELab color space is more uniform in visual perception is used, taking fully into account the luminance and chrominance information of the processed image, making the denoised images and human vision maintain better correlation. The experimental results have demonstrated the effectiveness of the algorithm from aspects of the subjective quality and the objective quality. This method is very helpful to further processing and application of downhole images.

\section{Conflict of Interests}

The authors declare that there is no conflict of interests regarding the publication of this paper.

\section{Acknowledgments}

This work is supported by advancing projects of the industrialization of scientific research achievements in Universities of Jiangsu Province (Grant JHB2012-36) and the science and technology fund of the Ministry of Housing and Urban-Rural Development of China (Grant 2013-K2-5).

\section{References}

[1] P. M. Narendra, "A separable median filter for image noise smoothing," IEEE Transactions on Pattern Analysis and Machine Intelligence, vol. 3, no. 1, pp. 20-29, 1981.

[2] J.-M. He, Q. Li, and Y. Ming, "Realization and performance test of improved median filter," Computer Systems \& Applications, vol. 18, no. 8, pp. 172-174, 2009.

[3] W. F. Darsow and E. T. Olsen, "Characterization of idempotent 2-copulas," Note di Matematica, vol. 30, no. 1, pp. 147-177, 2010.

[4] J. J. Li, H. Fan, and Y. Wang, "Image denoising algorithm based on dyadic contourlet transform," Applied Mechanics and Materials, vol. 40-41, pp. 591-597, 2011.

[5] G. Hernandez-Gomez, R. E. Sanchez-Yanez, V. Ayala-Ramirez, and F. E. Correa-Tome, "Natural image segmentation using the CIELab space," in Proceedings of the 19th International Conference on Electronics Communications and Computers (CONIELECOMP '09), pp. 107-110, February 2009.

[6] G. Cui, M. R. Luo, B. Rigg, G. Roesler, and K. Witt, "Uniform colour spaces based on the DIN99 colour-difference formula," Color Research and Application, vol. 27, no. 4, pp. 282-290, 2002.

[7] D.-H. Shin, R.-H. Park, S. Yang, and J.-H. Jung, "Blockbased noise estimation using adaptive Gaussian filtering," IEEE Transactions on Consumer Electronics, vol. 51, no. 1, pp. 218-226, 2005.

[8] S. Gao, C. Li, and D. Bi, "Image enhancement algorithm based on NF-ICM," Chinese Optics Letters, vol. 8, no. 5, pp. 474-477, 2010.

[9] A. Saffor, A. R. Ramli, and K.-H. Ng, "A comparative study of image compression between JPEG and wavelet," Malaysian Journal of Computer Science, vol. 14, no. 1, pp. 39-45, 2001.

[10] R. R. Coifinan and D. L. Donoho, "Translation-invariant denoising," in Wavelet in Statistics, vol. 103 of Lecture Notes in Statistics, pp. 125-150, Springer, New York, NY, USA, 1994.

[11] A. G. Bruce and H.-Y. Gao, "WaveShrink with firm shrinkage," Statistica Sinica, vol. 7, no. 4, pp. 855-874, 1997.

[12] M. Jansen, M. Malfait, and A. Bultheel, "Generalized cross validation for wavelet thresholding," Signal Processing, vol. 56, no. 1, pp. 33-44, 1997.

[13] L. Sendur and I. W. Selesnick, "Bivariate shrinkage functions for wavelet-based denoising exploiting interscale dependency," IEEE Transactions on Signal Processing, vol. 50, no. 11, pp. 27442756, 2002.

[14] C. S. Lee and Y. H. Kuo, Fuzzy Techniques in Image Processing, vol. 52, Springer, New York, NY, USA, 2000.

[15] C. S. Lee, Y. H. Kuo, and P. T. Yu, "Weighted fuzzy mean filters for image processing," Fuzzy Sets and Systems, vol. 89, pp. 15$180,1997$. 
[16] S. H. Wang, L. N. Wu, Y. D. Zhang et al., "Ant colony algorithm used for bankruptcy prediction.", in Proceedings of the 2nd International Symposium on Information Science and Engineering (ISISE '09), pp. 137-139, Shanghai, China, 2009.

[17] D. S. Levine and M. Aparicio IV, Eds., Neural Networks for Knowledge Representation and Inference, Psychology Press, 2013.

[18] S. Yuying and L. Jingjing, "A semi-implicit image denoising algorithm in matrix form," Journal of Xuzhou Institute of Technology (Natural Sciences Edition), vol. 27, no. 4, 2012. 


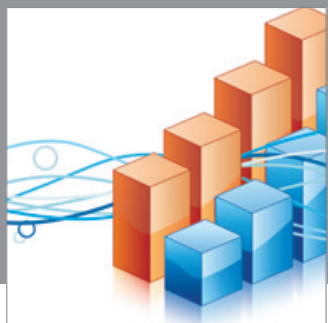

Advances in

Operations Research

mansans

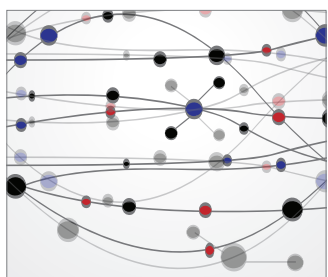

The Scientific World Journal
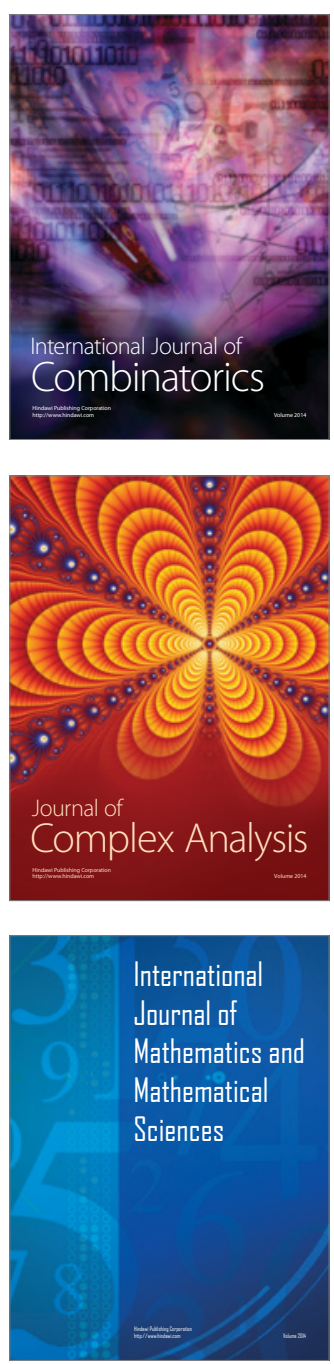
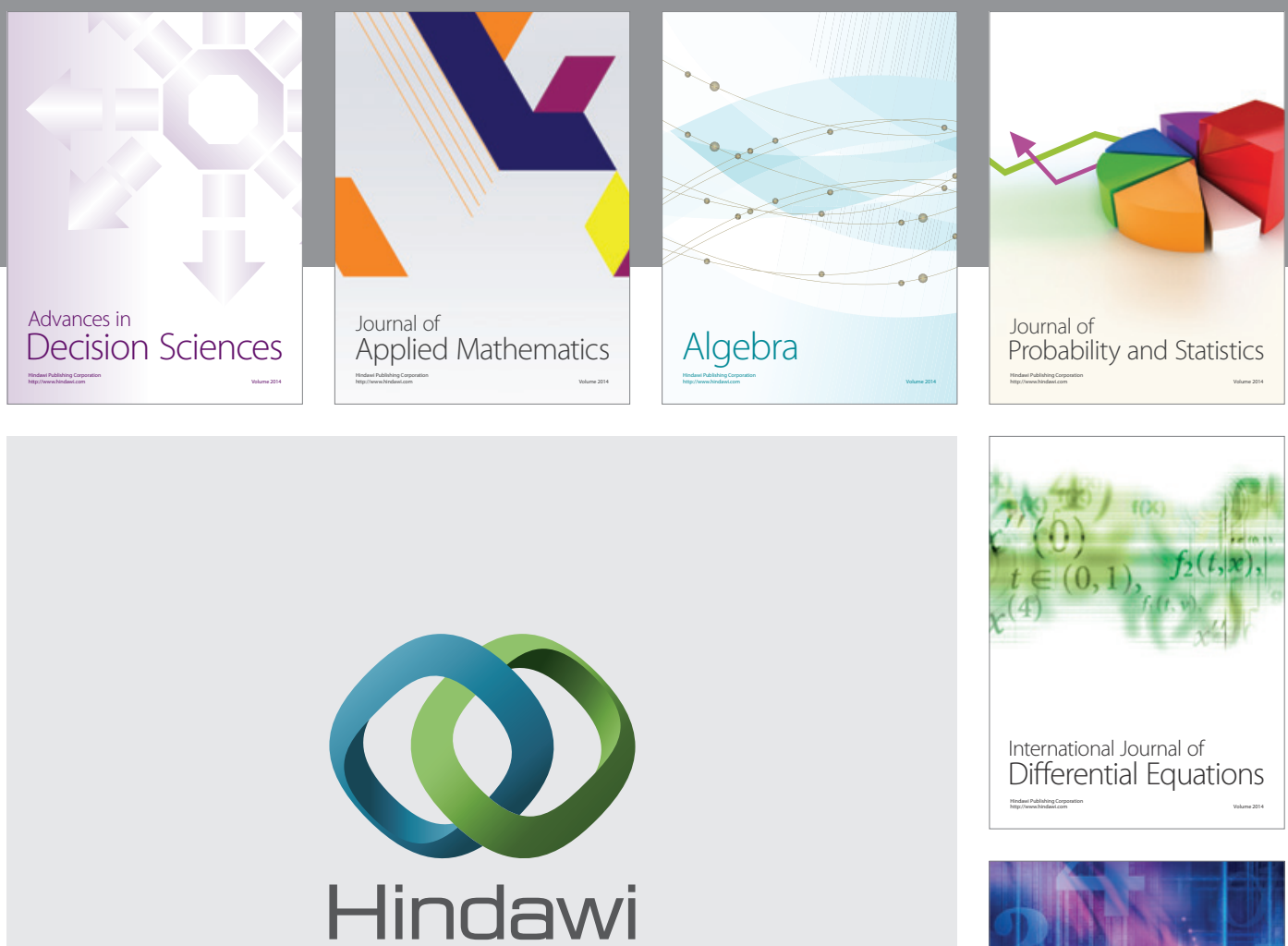

Submit your manuscripts at http://www.hindawi.com
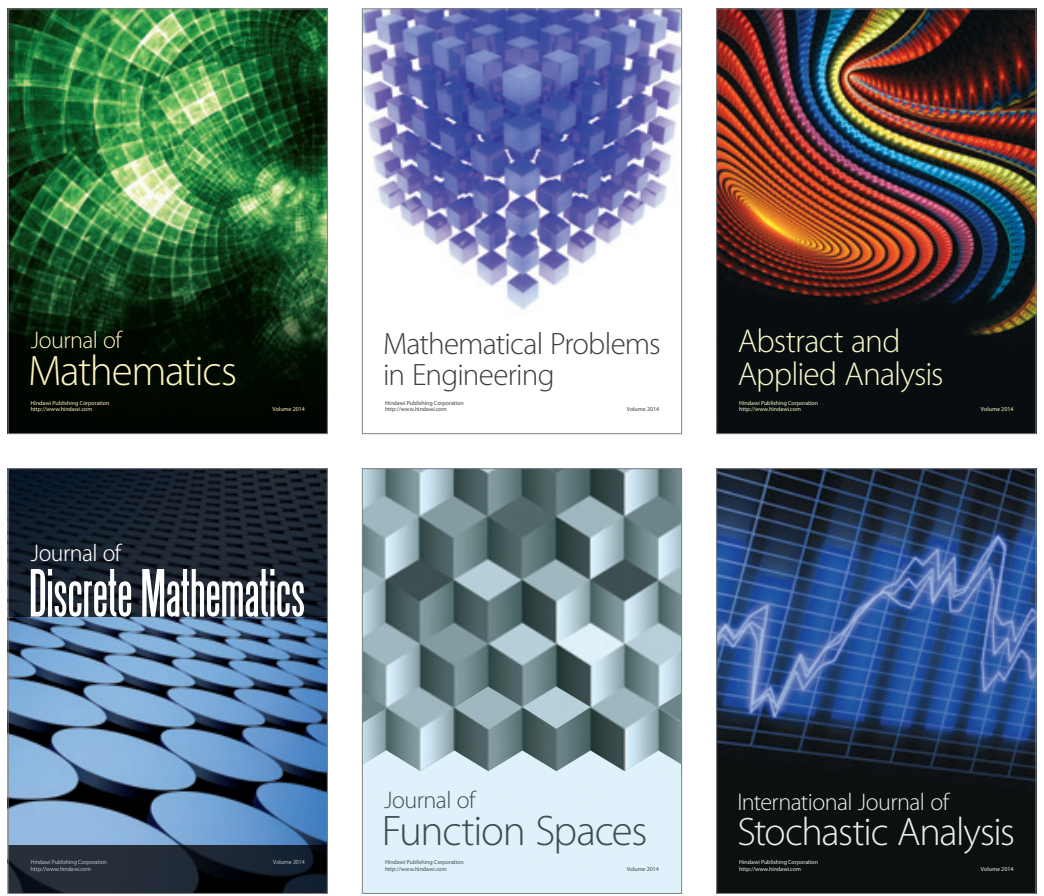

Journal of

Function Spaces

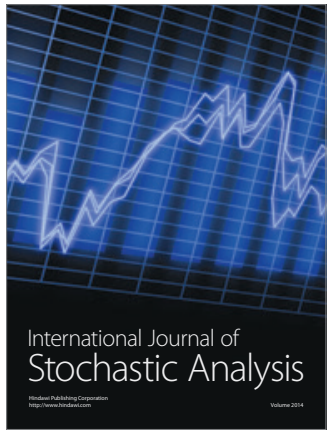

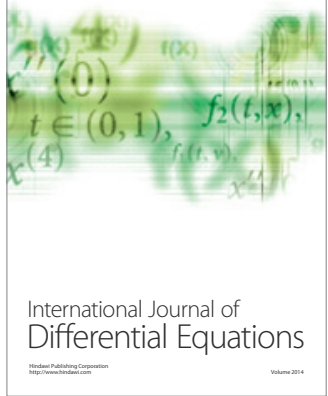
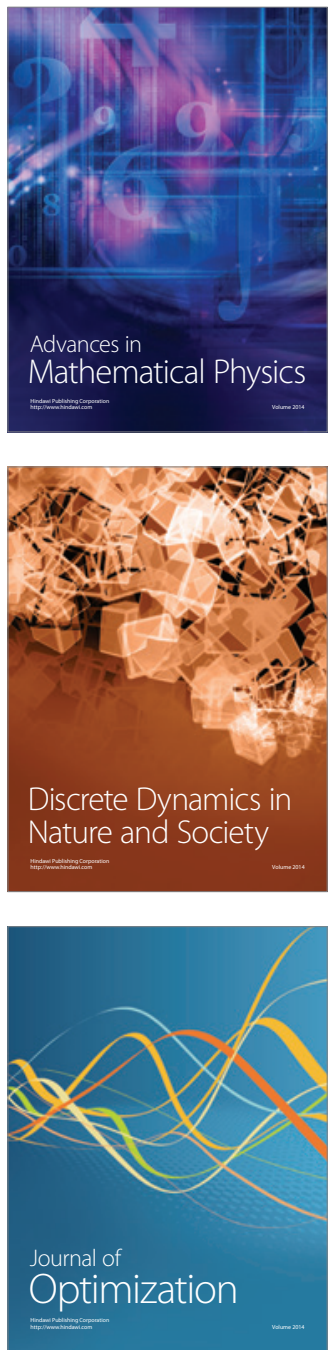